\title{
Structure of intumescent epoxy composites with expandable graphite
}

\author{
Alexandr Evstigneev ${ }^{1}$, Vladimir Smirnov ${ }^{1, *}$ and Vitaliy Gladkikh ${ }^{1}$ \\ ${ }^{1}$ Moscow State University of Civil Engineering, Yaroslavskoye hw. 26, 129337, Moscow, Russia
}

\begin{abstract}
Currently, thermoset-based polymer matrix composites are widely used for production of fire protective coatings. Intumescent properties of such coatings are highly dependent on composition. Expandable graphite is a prospective intumescent admixture; unfortunately, admixture of expandable graphite alone can lower the integrity of char residue down to unacceptable levels. The objective of the present work is to present the method that can be used for elimination of the mentioned drawback. The limiting values of expandable graphite in composite with two different admixtures - ammonium polyphosphate and ceramic cenospheres - were determined by means of study the structure of both intumescent epoxy composites and char residues of such composites. It was revealed that admixture of ceramic cenospheres leads to notable improvement of integrity of char residue. This improvement is mostly due to features of heat transfer in material with cenospheres which are characterized by low particle size and, at the same time, low heat conductance.
\end{abstract}

\section{Introduction}

Constant complication of architectural forms requires extensive use of constructional steel [1-3]. One of the primary problems is that at high temperatures (open fire) elastic modulus of steel lowers down to unacceptable levels; this is the primary reason of failures of constructions during fire [4-6].

Efficient prevention of early failure of constructions during fire can be implemented by means of special type of coating, namely intumescent coating. During fire, intumescent coating transforms into thick porous layer (char residue) with low thermal conductivity. This prevents quick heating of underlying steel and prolongs the time before critical failure of constructions [7-10]. This type of fire protection is often referred to as "passive".

The primary choice during R\&D of intumescent coating is the type of binder (it terms of theory of composites - "matrix material"). Rheological properties of matrix material determine the technology of coating and affect the value of maximal thickness that can be achieved during single pass layer coating. Dependence between temperature and elastoplasic properties (glass transition or melting point temperature), together with carbonization temperature of matrix govern the choice of intumescent and other functional dispersed phases.

\footnotetext{
*Corresponding author: smirnov@nocnt.ru
} 
Composites with epoxy matrices are widely used in construction. Such composites often characterized by high strength, low weathering and high durability under severe environmental conditions. Important advantage of thermosetting matrix is the possibility to control glass transition temperature by means of thermal processing. Thus, epoxy resins can be considered as prospective matrix materials for passive fire protection of steel [11-16].

Total volumetric content of dispersed phases affects rheological properties of composition and, due to this, technology and maximal thickness of coating. For epoxies, this content should be about $1 / 2$. Till now, heat conductance of matrix and dispersed phases usually was not taken into account. As it will be shown below, in some cases values of heat conductance are of extreme importance.

Usually, composition for intumescent coating includes special dispersed phases, e.g. ammonium polyphosphate (APP) and pentaerythritol [7, 17]. Relatively new intumescent admixture is the expandable graphite (EG) that can be produced by treatment of flake graphite with mixture of acids and some oxidizing agents [18-21]. Expandable graphite can also improve many properties (barrier properties, resistance to aggressive mediums and resistance to weathering) of coating under normal operation.

The objective of the present work is to study and optimize the structure of char residues of epoxy composite that includes APP and EG as intumescent dispersed phases.

\section{Materials and methods}

As matrix material and hardener we have used DER-330 (Dow Chemical, [22]) epoxy resin and 9210P epoxyamine complex, respectively. Mass ratio of hardener in epoxy-hardener composition was 0.65 .

There were two types of intumescent admixtures: APP and EG with average flake diameter of $0.3 \mathrm{~mm}$ and $99 \%$ purity. Aluminum hydroxide (AH, RU GOST 11841) and hollow fly ash aluminosilicate (ceramic) cenospheres (ASC) with average size $100 \mathrm{mkm}$ and reduced density $600 \mathrm{~kg} / \mathrm{m}^{3}$ were used as non-intumescent functional fillers.

Two series of experiments were conducted. First series was carried out to in accordance with 7-point experimental design that is shown on Figure 1a; the goal was to estimate the expansion factor of composites with APP, EG and AH. Second series was performed in accordance with 9-point " $2^{2}+5$ " central composite experimental design (Figure 1b); the goal was to prove hypothesis about reason of low integrity of char residue.

For both series of experiments, total volumetric content of dispersed phases was 0.5 . In first series, volumetric contents of EG, APP and AH were varied in range 0...0.5 (Figure 1). In second series, volumetric content of EG and APP was varied in range 0.032...0.88 $\left(0.04 \ldots 0.08\right.$ for $2^{2}$ full factorial part) and $0.079 \ldots 0.221\left(0.1 \ldots 0.02\right.$ for $2^{2}$ full factorial part $)$, respectively. Consequently, volumetric content of CC was varied in range $0.219 \ldots 0.361$ $\left(0.22 \ldots 0.36\right.$ for $2^{2}$ full factorial part).

The samples were prepared as follows: epoxy resin was combined with hardener; mix homogenized for 5 minutes; epoxy-hardener composition was combined with dispersed phases; mix homogenized for 5 minutes; composition filled into molds and hardened for 24 hours. All samples are cubes with $10 \mathrm{~mm}$ edge.

Optical images were obtained with DSLR camera. X-ray images were obtained with SkyScan 1173 tomograph (Bruker Optik). "Darktable" software was used for images processing (brightness-contrast adjustment, noise reduction and elimination of global linear gradient of brightness). 
a)

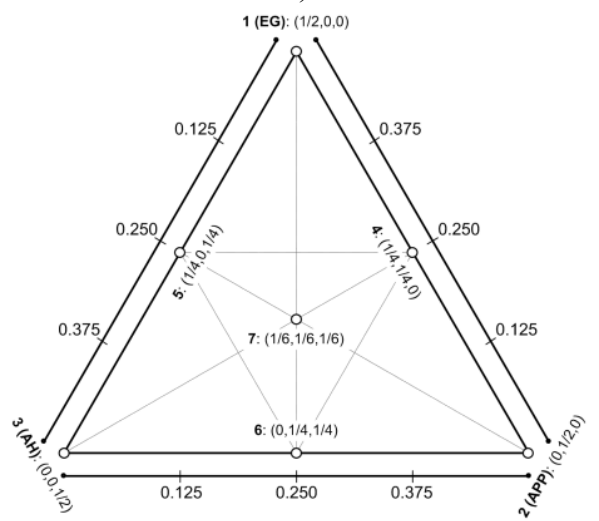

b)

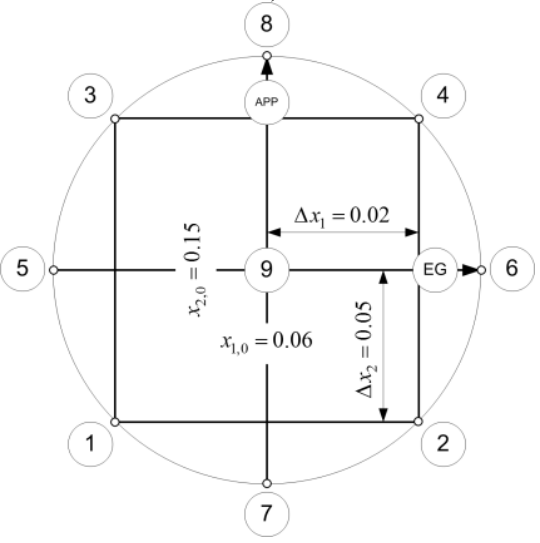

Fig. 1. Experimental design for estimation of expansion factor (a) and optimization of integrity of char residue (b).

To study the expansion factor and structure of char residue, samples were glued by epoxy on metal substrate and that processed in muffle furnace. There were three temperature ramps: from room temperature to $350{ }^{\circ} \mathrm{C}$ in 30 minutes; exposure at $350{ }^{\circ} \mathrm{C}$ for 10 minutes; cooling down to room temperature for 2 hours. To estimate the expansion factor, three mutually perpendicular dimensions $a, b$ and $c$ of expanded samples were interpreted as two axes and semi-axis of triaxial ellipsoid; than, volume of expanded sample was calculated as $\pi \mathrm{abc} / 3$ (Figure 2).

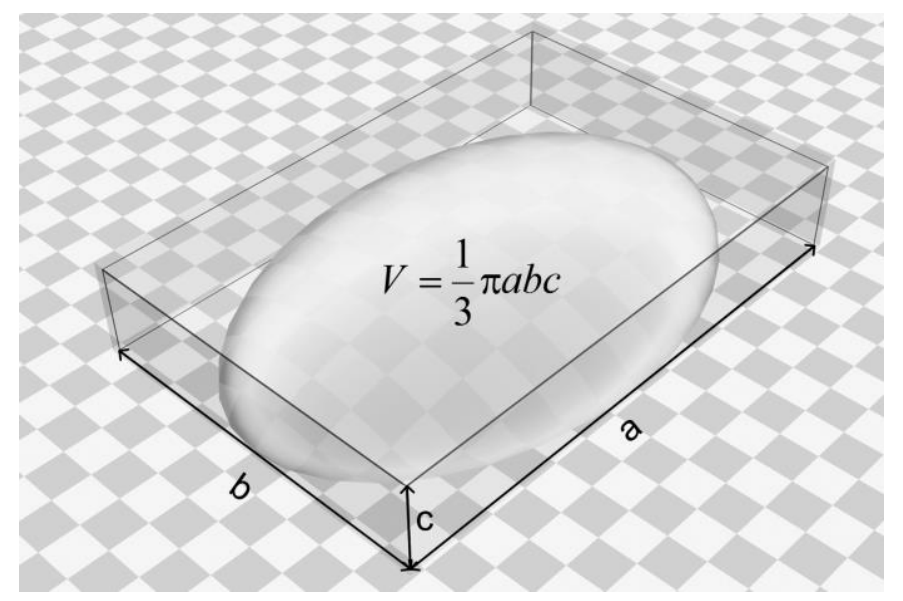

Fig. 2. Estimation of volume of expanded char residue.

Integrity of char residue was estimated in [0..1] scale, where " 0 " corresponds to residue in form of fine powder and "1" corresponds to tight residue that has not exfoliated from metal substrate.

For regression analysis and ternary plots the software was used. Function plots were made with gnuplot. 


\section{Results and Discussion}

To visualize the inner structure of samples during first series of experiments, small amounts of lead red $\left(\mathrm{Pb}_{3} \mathrm{O}_{4}\right)$ were added to the compositions. X-ray images of two resulting samples (before processing in furnace) are presented on Figure 3.

a)

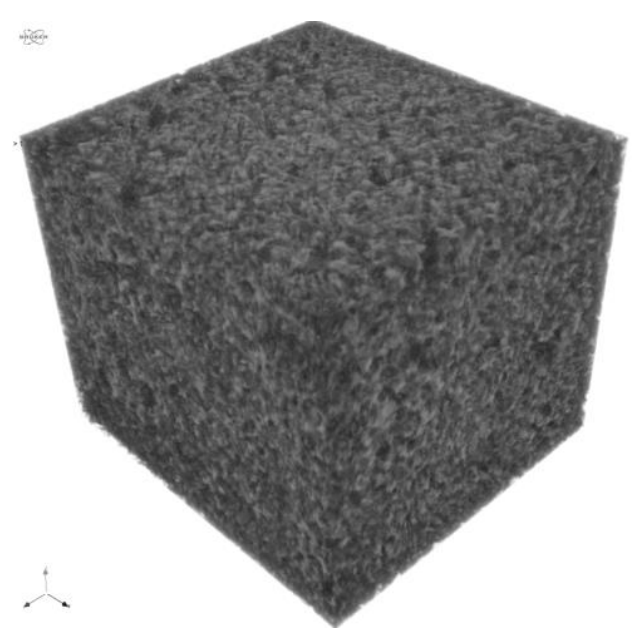

b)

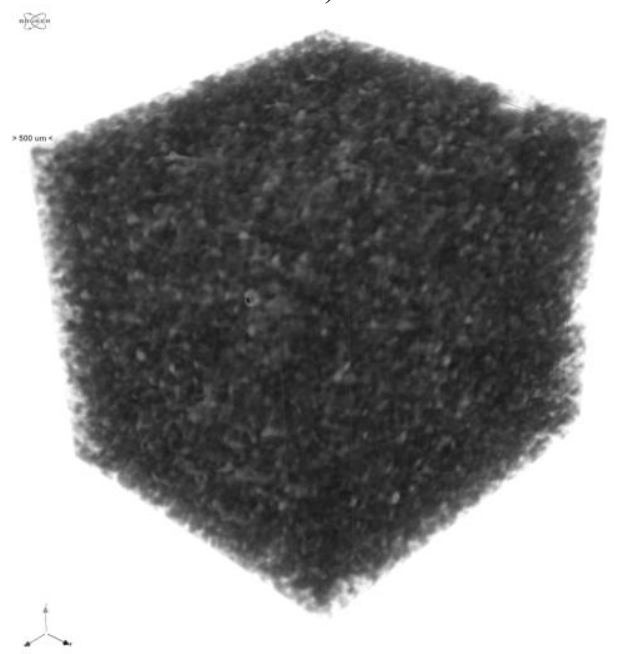

Fig. 3. X-ray images of samples from first series of experiments: point 1, sample with EG only (a) and point 2, sample with APP only (b).

As it follows from Figure 3, despite the bad thermodynamic compatibility between APP and matrix material, both EG and APP can easily be homogenized within the structure of sample.

During first series of experiments, two regression models were obtained:

- expansion factor:

$$
X f=32.5 x_{1}+5.12 x_{2}+1.18 x_{3}+23.72 x_{1} x_{2}+20.48 x_{1} x_{3}+2.57 x_{2} x_{3}+151.76 x_{1} x_{2} x_{3} ;
$$

- integrity of char residue:

$$
I r=0.50 x_{2}+1.00 x_{3}-0.20 x_{1} x_{2}-0.8 x_{1} x_{3}-0.2 x_{2} x_{3}-0.45 x_{1} x_{2} x_{3},
$$

where $x_{i}$ - volumetric contents of EG, APP and AH, normalized to [0...1] range.

Contour plots of expansion factor and integrity of char residue (regression models (1) and (2)) are presented on Figure 4.

As it follows from (1), (2) and Figure 4a, volumetric content of EG is the primary factor which affects expansion of char residue. Value of expansion factor in the first experimental point $(1,0,0)$ which corresponds to filling by EG alone, is six times higher than in second point (single APP as a filler) and 28 times higher than in third point (single AH as a filler).

For the first edge of factor space $\left(x_{3}=0\right)$ the model of expansion factor becomes

$$
X f=32.5 x_{1}+5.12 x_{2}+23.72 x_{1} x_{2},
$$

or, taking into account that $x_{2}=1-x_{1}$ :

$$
X f=5.12+51.1 x_{1}-23.72 x_{1}^{2} .
$$

For the third edge of factor space $\left(x_{2}=0\right)$ the model of expansion factor becomes 


$$
X f=1.18+51.8 x_{1}-20.48 x_{1}^{2} .
$$

a)

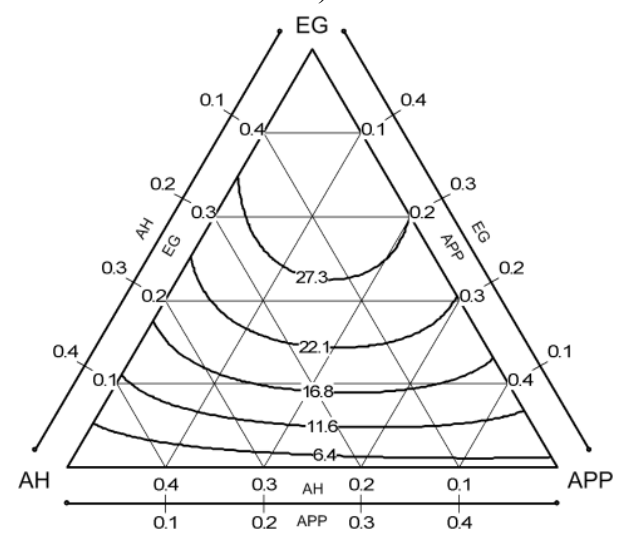

b)

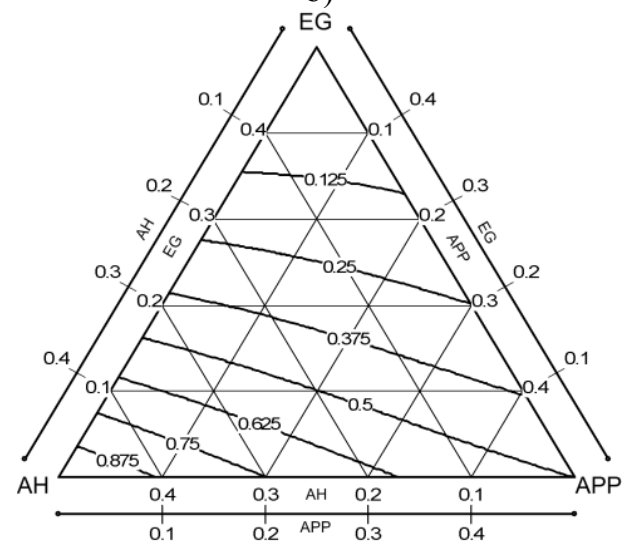

Fig. 4. Expansion factor (a) and integrity of char residue (b).

Dependencies (4) and (5) between volumetric content of EG and expansion factor, together with corresponding rates of change (first derivatives), are presented on Figure 5.

a)

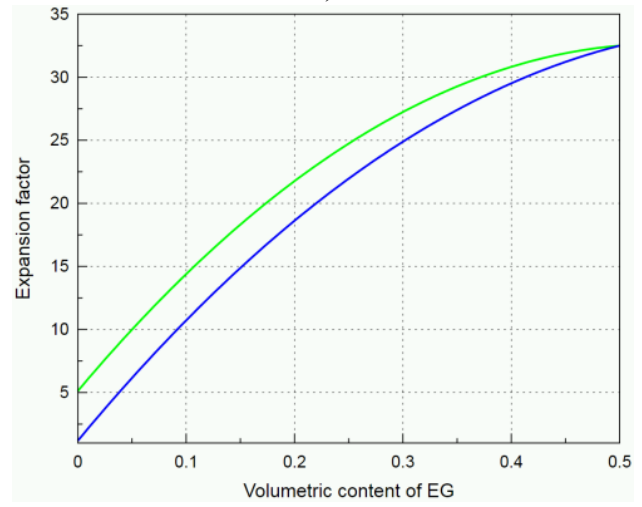

b)

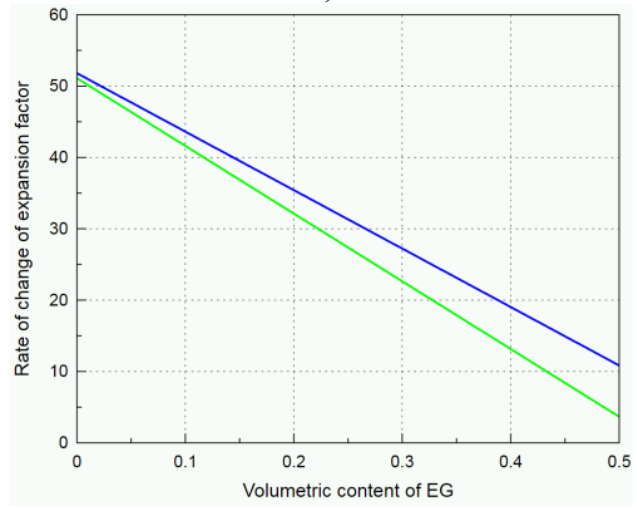

Fig. 5. Dependencies between volumetric content of $E G$ and expansion factor (a); corresponding rates of change (b). Green lines correspond to $x_{3}=0$, blue ones - to $x_{2}=0$.

As it follows from Figure 5, near the first point of experimental design (EG only; rightmost areas on Figure 5) the expansion factor varies very slowly; in other words, small amounts of other dispersed phases, be it APP or AH, do not affect value expansion factor. The leftmost areas are on Figure 5 are more interesting: while the values of expansion factor are small, the rates are an order of magnitude higher. Therefore, small variation of EG content is enough for efficient control of intumescent properties.

Two questions are immediately raised because of these consistent patterns:

- do we need the other dispersed phases except the EG?

- can the AH be exchanged by another functional phase without notable loss of intumescent effect?

The answers to both questions are "yes". Though the value of expansion factor is highest in first point of experimental design, the integrity of char residue (Figure 6) is unacceptable (Figure 4b). 


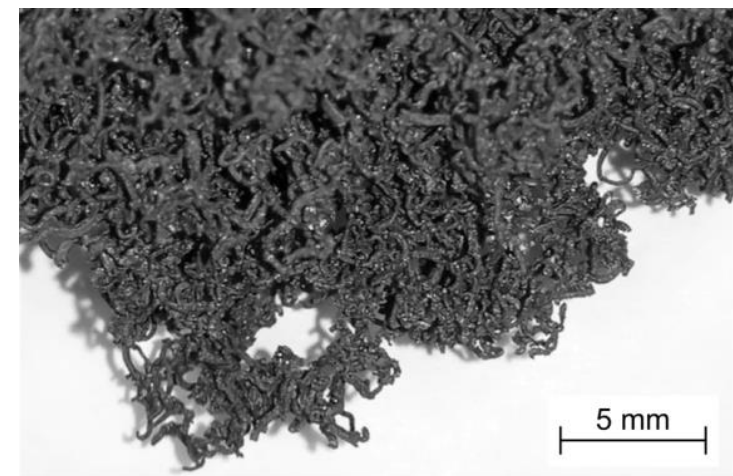

Fig. 6. Char residue of composite with single EG as dispersed phase.

At the same time, integrity can be significantly improved (Figure 4b) if the EG content is lowered. And such a measure does not lead to high declination of intumescent properties (Figure 4a).

The AH was originally selected as a phase that lowers combustibility of composite. For intumescent coating, combustibility is not essential; thus, taking into account low expansion factor (near 1) in third point of experimental design, $\mathrm{AH}$ can be freely exchanged to other functional dispersed phase.

Still, even in the proximity of third point of experimental design, integrity of char residue for composites with EG is too low for practical application. Complete exclusion of EG (second, lower edge of experimental design) is not an option because this phase is very efficient intumescent admixture.

The following hypothesis can be an explanation of the low integrity: destruction of the char residue is due to considerable difference in heat conductivity of matrix and EG. This difference leads to expansion of EG inside the matrix which is still not reached glass transition.

In the framework of the stated hypothesis there are two opposite methods that can be used for the improvement of integrity:

- to equalize the heat conductivity of matrix and EG by means of dispersed phase with high heat conductivity (fine metallic or flake graphite powder);

- to prevent quick heating of EG particles by means of dispersed phase with low heat conductivity.

The first method has many drawbacks from the practical point of view: any dispersed phase with high heat conductivity will inevitably disimprove the heat barrier properties of resulting char residue. Thus, to prove the hypothesis, the second method can be tested.

In accordance to the stated above, $\mathrm{AH}$ was exchanged by fine dispersed phase with low heat conductivity - CC. Since the entire factor space (Figure 1a) is of low interest, only small subset of factor space is studied (Figure 1b); the relation between full set (first series of experiments) and subset (second series) is illustrated by Figure 7.

It can easily be seen from Figure 7 that selected area of interest corresponds to the area in which intumescent properties are in high dependence on the volumetric content of EG (leftmost areas on Figure 5).

Numerous properties were studied during second series of experiments, and plenty of regression models were derived; but considering the purpose of the present work, all that has to be done is to examine the structure of char residue. Optical images of char residue for point 2 (high content of EG, low content of APP) and point 3 (the opposite) are presented on Figure 8. 


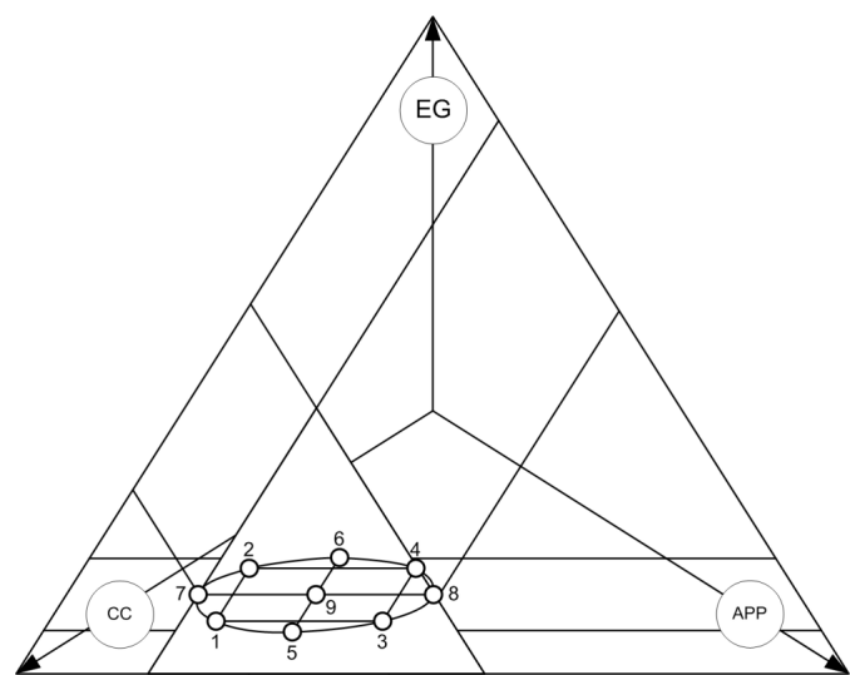

Fig. 7. Relation between full range of control variables (Figure 1a) and subset (Figure 1b) that is studied during second series of experiments.

a)

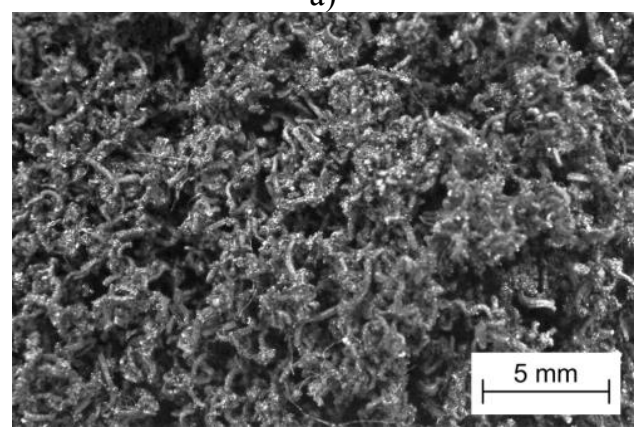

b)

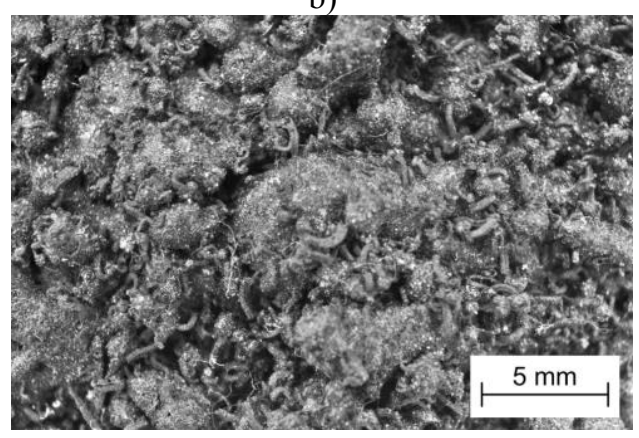

Fig. 8. Char residues of composites with CC: EG prevails (a), APP prevails (b).

It is evident from Figure 8 that integrity of char residues is improved notably. Even for the composite with high content of EG, char residue neither falls to pieces, nor exfoliates from metal substrate. Thus, the experimental results do not contradict the above hypothesis.

\section{Summary}

The novelty of the present work is mostly in hypothesis about the reason of previously known fact that high volumetric content (approximately above 5\%) of expandable graphite leads to breaking of char residue during coking of epoxy composite.

The relation between heat conductivities of EG and matrix was taken as a key point of the proposed explanation. It was shown that experimental results do not contradict the explanation and, simultaneously, the efficient method that may be used to improve the integrity of char residue was offered.

The practical value of the work is due to importance of passive fire protection of steel.

This work is supported by grant of Russian Federation President SP-2627.2016.1. 


\section{References}

1. C.G. Salmon, Steel Structures: Design and Behavior (Prentice Hall, New Jersey, 2008)

2. K.S. Sai Ram, Design of Steel Structures (Pearson Education, Chennai, 2010)

3. N.R. Baddoo, Journal of Constructional Steel Research 64, 1199-1206 (2008)

4. T.C.H Liu, M.K. Fahad, J.M. Davies, Journal of Constructional Steel Research 58, 1211-1230 (2002)

5. F. Wald, L. Simões da Silva, D.B. Moore, T. Lennon, M. Chladná, A. Santiago, M. Beneš, L. Borges, Fire Safety Journal 41, 509-522 (2006)

6. M. Feng, Y.C. Wang, J.M. Davies, Fire Safety Journal 38, 365-394 (2003)

7. M. Jimenez, S. Duquesne, S. Bourbigot, Thermochimica acta 449, 16-26 (2006)

8. M. Jimenez, S. Duquesne, S. Bourbigot. Surface and coatings Technology 201, 979987 (2006)

9. M. Jimenez, S. Bellayer, B. Revel, S. Duquesne, S. Bourbigot, Industrial \& Engineering Chemistry Research 52, 729-743 (2013)

10. B.K. Cirpici, Y.C. Wang, B.D. Rogers, S Bourbigot, Polymer Engineering \& Science 56, 798-809 (2016)

11. J.S. Wang, Y. Liu, H.B. Zhao, J. Liu, D.Y. Wang, Y.P. Song., Y.Z. Wang, Polymer Degradation and Stability 94, 625-631 (2009)

12. C. Gérard, G. Fontaine, S. Bourbigot, Polymers for Advances Technologies 22, 10851090 (2011)

13. C. Gérard, G. Fontaine, S. Bellayer, S. Bourbigot, Polymer Degradation and Stability 97, 1366-1386 (2012)

14. M. Gomez-Mares, A. Tugnoli, G. Landucci, F. Barontini, V. Cozzani, Journal of Analytical and Applied Pyrolysis 97, 99-108 (2012)

15. V. Smirnov, E. Korolev, MATEC Web of Conferences 193, 03028 (2018)

16. A. Evstigneev, V. Smirnov, E. Korolev, MATEC Web of Conferences 251, 01033 (2018)

17. S. Bourbigot, S. Duquesne, Journal of Materials Chemistry 17, 2283-2300 (2007)

18. Z. Wang, E. Han, W. Ke, Corrosion Science 49, 2237-2253 (2007)

19. S. Ullah, F. Ahmad, P.S.M. Megat-Yusoff, N.H. Azmi, Journal of Applied Sciences 11, 1630-1635 (2011)

20. S. Ullah, F. Ahmad, A.M. Shariff, M.A. Bustam, AIP Conference Proceedings 1621, 335 (2014)

21. Y. Li, S. Zou, J. Zhou, Y. Chen, H. Zou, M. Liang, W. Luo, Journal of Applied Polymer Science 131, 39885 (2014)

22. J. Asante, F. Modiba, B. Mwakikunga, International Journal of Polymer Science 2016, 1792502 (2016) 